\title{
THE SHAPE OF LOW ENERGY CONFIGURATIONS OF A THIN ELASTIC SHEET WITH A SINGLE DISCLINATION
}

\author{
HEINER OLBERMANN
}

\begin{abstract}
We consider a geometrically fully nonlinear variational model for thin elastic sheets that contain a single disclination. The free elastic energy contains the thickness $h$ as a small parameter. We give an improvement of a recently proved energy scaling law, removing the next-to leading order terms in the lower bound. Then we prove the convergence of (almost-)minimizers of the free elastic energy towards the shape of a radially symmetric cone, up to Euclidean motions, weakly in the spaces $W^{2,2}\left(B_{1} \backslash B_{\rho} ; \mathbb{R}^{3}\right)$ for every $0<\rho<1$, as the thickness $h$ is sent to 0 .
\end{abstract}

\section{INTRODUCTION}

1.1. Setup and previous work. The present article continues a program [28, 31, 32] to explore thin elastic sheets with a single disclination from the variational point of view. The free energy that we consider consists of two parts: First, the non-convex membrane energy, that penalizes the difference between the metric that is induced by the deformation and the reference metric, which is the metric of the (singular) cone. Second, the bending energy, which penalizes curvature. The bending energy contains a factor $h^{2}$, where the small parameter $h$ is to be thought of as the thickness of the sheet (see equation (1) below for the definition). Choosing the cone as configuration, one gets infinite energy: While the membrane term vanishes, the bending energy is infinite for this choice. Energetically, there is a competition of the membrane and the bending terms; neither will vanish for configurations of low energy.

Intuitively, it seems quite clear how configurations of low energy should look like: They should be identical to the cone far away from the disclination, and near the disclination, there should be some smoothing of the cone, at a scale $h$ (the only length scale in the problem). For such configurations, one gets an energy of $C^{*} h^{2} \log \frac{1}{h}$ plus terms of order $h^{2}$, where $C^{*}$ is an explicitly known constant, see Lemma 2 below. It is natural to conjecture that such a scaling behavior should indeed hold true for minimizers. However, a proof of an ansatz-free lower bound with the same scaling is much more difficult than the straightforward construction for the upper bound. In the literature, lower bounds for this setting have been ansatz based [23, 36, 39], or have assumed radial symmetry [28].

The idea underlying the recent proofs of ansatz-free lower bounds [31, 32] is to control the Gauss curvature (or a linearization thereof) by interpolation between the membrane and the bending term energy. The control over the Gauss curvature allows for a certain control over the Gauss map (or the deformation gradient). This information in turn yields lower bounds for the bending energy, using an inequality of Sobolev/isoperimetric type. For the corresponding result from [32] see equation (2) below. This lower bound does not

Date: October 8, 2018. 
quite achieve the conjectured scaling behavior, in that there exist next-to leading order terms $O\left(h^{2} \log \log \frac{1}{h}\right)$ which are not present in the upper bound.

Here, we are going to improve the results from [32] in two ways: First, we give an improved lower bound for the elastic energy, which proves the conjecture that the minimum of the energy is given by $C^{*} h^{2} \log \frac{1}{h}+O\left(h^{2}\right)$. The observation that allows for this improvement is that it is unnecessary to use interpolation to control Gauss curvature and Gauss map (or rather, linearized Gauss curvature and deformation gradient). It is enough to use the membrane energy alone to obtain the necessary control, and make more efficient use of the Sobolev/isoperimetric inequality.

Second, we use this improved lower bound to show a statement about the shape of configurations that satisfy the energy bounds. We prove that (almost-)minimizers converge to the conical deformation, up to Euclidean motions. It is remarkable that that much information about deformations of small energy can be obtained, considering that we are dealing with a highly non-convex variational problem. Hitherto, such results had only been achieved for situations in which the energy scales like $O\left(h^{2}\right)$ or less [12, 15, 33]. The results of these papers will also play an important role in our proof.

1.2. Statement of results. Let $B_{1}:=\left\{x \in \mathbb{R}^{2}:|x|<1\right\}$ be the sheet in the reference configuration. The singular cone may be described by the mapping $y^{\Delta}: B_{1} \rightarrow \mathbb{R}^{3}$,

$$
y^{\Delta}(x)=\sqrt{1-\Delta^{2}} x+\Delta|x| e_{3} .
$$

Here, $0<\Delta<1$ is the height of the singular cone, and is determined by the deficit of the disclination at the origin. The reference metric on $B_{1}$ is given by

$$
\begin{aligned}
g_{\Delta}(x) & =D y^{\Delta}(x)^{T} D y^{\Delta}(x) \\
& =\left(1-\Delta^{2}\right) \hat{x} \otimes \hat{x}+\Delta^{2} \hat{x} \otimes \hat{x} \\
& =\operatorname{Id}_{2 \times 2}-\Delta^{2} \hat{x}^{\perp} \otimes \hat{x}^{\perp},
\end{aligned}
$$

where $\hat{x}=x /|x|$ and $\hat{x}^{\perp}=\left(-x_{2}, x_{1}\right) /|x|$. The induced metric of a deformation $y \in$ $W^{2,2}\left(B_{1} ; \mathbb{R}^{3}\right)$ is

$$
g_{y}=D y^{T} D y \text {. }
$$

The free elastic energy $I_{h, \Delta}: W^{2,2}\left(B_{1} ; \mathbb{R}^{3}\right) \rightarrow \mathbb{R}$ is defined by

$$
I_{h, \Delta}(y)=\int_{B_{1}}\left(\left|g_{y}-g_{\Delta}\right|^{2}+h^{2}\left|D^{2} y\right|^{2}\right) \mathrm{d} \mathcal{L}^{2},
$$

where $\mathrm{d} \mathcal{L}^{2}$ denotes 2-dimensional Lebesgue measure. In the paper [32], we proved the existence of a constant $C=C(\Delta)>0$ such that

$$
2 \pi \Delta^{2} h^{2}\left(\log \frac{1}{h}-2 \log \log \frac{1}{h}-C\right) \leq \min _{y \in W^{2,2}\left(B_{1} ; \mathbb{R}^{3}\right)} I_{h, \Delta}(y) \leq 2 \pi \Delta^{2} h^{2}\left(\log \frac{1}{h}+C\right) .
$$

Our first aim in the present article is to improve the lower bound for the free elastic energy. The improvement consists in getting rid of the $\log \log \frac{1}{h}$ terms on the left hand side:

Theorem 1. There exist positive constants $C_{1}, C_{2}, C_{3}$ that only depend on $\Delta$ with the following property: First,

$$
2 \pi \Delta^{2} h^{2}\left(\log \frac{1}{h}-C_{1}\right) \leq \min _{y \in W^{2,2}\left(B_{1} ; \mathbb{R}^{3}\right)} I_{h, \Delta}(y) \leq 2 \pi \Delta^{2} h^{2}\left(\log \frac{1}{h}+C_{2}\right)
$$


for all small enough $h>0$. Furthermore, if y satisfies

$$
I_{h, \Delta}(y) \leq 2 \pi \Delta^{2} h^{2}\left(\log \frac{1}{h}+C_{2}\right)
$$

then

$$
\begin{aligned}
& \int_{B_{1} \backslash B_{R}}\left|D^{2} y\right|^{2} \mathrm{~d} \mathcal{L}^{2} \leq 2 \pi \Delta^{2} \log \frac{1}{R}+C_{3} \quad \text { for all } R \in(2 h, 1), \\
& \int_{B_{1}}\left|g_{y}-g_{\Delta}\right|^{2} \mathrm{~d} \mathcal{L}^{2} \leq C_{3} h^{2} .
\end{aligned}
$$

As a consequence of Theorem 1 , we will be able to prove convergence of (almost)-minimizers of the functional (1) towards the singular cone as $h \rightarrow 0$ :

Theorem 2. Let $y^{h} \in W^{2,2}\left(B_{1} ; \mathbb{R}^{3}\right)$ be a sequence with $I_{h, \Delta}\left(y^{h}\right) \leq 2 \pi \Delta^{2} h^{2}\left(\log \frac{1}{h}+C_{2}\right)$. Then up to Euclidean motions, we have for every $0<\rho<1$,

$$
y^{h} \rightarrow y^{\Delta} \quad \text { in } W^{2,2}\left(B_{1} \backslash B_{\rho} ; \mathbb{R}^{3}\right) .
$$

1.3. Scientific context. In the proof of Theorem 1 we show a certain focusing of the elastic energy near the disclination. Phenomena with such elastic energy focusing are also observed in many other settings. In particular, crumpled elastic sheets display networks of vertices and ridges. The investigation of these "sharp" structures in the physics community started in the mid-1990's. For a historical account and an exhaustive list of references see the very recommendable overview article by Witten [38]. There has been quite some activity in the analysis of ridge-like structures in particular, see [11, 19, 24, 25, 26, 37]. Energy focusing in conical shapes has been investigated in [3, 6, 7, 8]. Disclinations in thin elastic sheets are particularly interesting as a modeling device for icosahedral elastic structures. This is a popular model for virus capsids [23, 36] or carbon nanocones [35], the structure one obtains when inserting a single five-valent vertex into a graphene sheet (of otherwise six-valent vertices). The disclinations are located at the vertices of the elastic icosahedra.

In the mathematical literature on thin elastic sheets, there have been two strands of investigation: On the one hand, there are the rigorous derivations of elastic plate models from three-dimensional finite elasticity by means of $\Gamma$-convergence (see [12, 13, 21]). On the other hand, there has been quite some effort to investigate the qualitative properties of low-energy states in the variational formulation of elasticity, obtained through an analysis of the scaling of the free elastic energy with respect to the relevant parameters in the model, see e.g. [1, 2, 4, 18]. The present paper belongs of course to the latter group. In more detail, rigorous scaling laws similar to the ones we prove here have been derived for a single fold [10] and for the so-called $d$-cone [5, 29]. The variational problems considered in these references however are of a very special kind: The constraints on the shape of the elastic sheet are quite restrictive, and the lower bounds use these constraints in an essential way (see [32] for a detailed discussion). This is not the case for our setting, whence our method of proof, which we have developed in [31, 32] and which we refine here, is completely different. 
1.4. Connection to convex integration and rigidity results. The Nash-Kuiper Theorem [20, 30] states that given a two-dimensional Riemannian manifold $(M, g)$, a short ${ }^{1}$ immersion $y_{0}: M \rightarrow \mathbb{R}^{3}$, and $\varepsilon>0$, there exists an isometric immersion $y_{1} \in C^{1}\left(M ; \mathbb{R}^{3}\right)$ such that $\left\|y_{1}-y_{0}\right\|_{C^{0}}<\varepsilon$. This is relevant in our context, since the leading order term in the energy (1) measures the distance of the deformation $y$ from an isometric immersion with respect to the target metric $g_{\Delta}$. By the Nash-Kuiper Theorem, there exists a vast amount of deformations $y$ that have arbitrarily small membrane energy. A priori, these are all good candidates for energy minimization. One needs a principle that shows that all of these deformations are associated with large bending energy. The energy scaling law from Theorem 1 shows that none of these maps can beat the upper bound construction energetically. Theorem 2 shows the "stronger" statement that maps with low energy cannot look anything like the approximations of $C^{1}$ isometric immersions that appear in the proof of the Nash-Kuiper Theorem.

The Nash-Kuiper result is an instance of convex integration, a concept that has been developed systematically by Gromov [14]. In particular, the theorem states that solutions to isometric immersion problems are highly non-unique if one requires only $C^{1}$-regularity. In stark contrast, there is the uniqueness in the Weyl Problem: Given a sufficiently smooth metric $g$ on $S^{2}$ with positive Gauss curvature, there exists a unique isometric immersion $y: S^{2} \rightarrow \mathbb{R}^{3}$ of $C^{2}$-regularity. Such uniqueness is often called rigidity. The dichotomy of convex integration versus rigidity also appears in other contexts, such as the MongeAmpère equation [22] and the incompressible Euler equation [9, 17].

Concerning the uniqueness of solutions in the Weyl problem, the proof is due to Pogorelov [34. In fact, he proved that solutions are unique up to Euclidean motions in the class of immersions of bounded extrinsic curvature. The latter is the class of immersions for which the pull-back of the volume form on $S^{2}$ under the Gauss map is a well defined signed Radon measure. For smooth maps, this is just the measure $K \mathrm{~d} A$, where $K$ is the Gauss curvature and $\mathrm{d} A$ the volume element. We see that control over the Gauss curvature excludes constructions in the style of Nash-Kuiper. This is also the basic concept underlying our proof (with the modification that we consider a linearized version of Gauss curvature). We believe that this hints at a link between questions about rigidity of surfaces and variational problems in the theory of thin elastic sheets.

Notation. For a closed line segment $\{a+t(b-a): t \in[0,1]\} \subset \mathbb{R}^{2}$, we write $[a, b]$. For a semi-closed line segment $\{a+t(b-a): t \in(0,1]\} \subset \mathbb{R}^{2}$, we write $(a, b]$. Throughout the text, we will assume the deficit of the disclination $0<\Delta<1$ to be fixed. A statement such as " $f \leq C g$ " is shorthand for "there exists a constant $C>0$ that only depends on $\Delta$ such that $f \leq C g$ ". The value of $C$ may change within the same line.

For $r>0$, we let $B_{r}=\left\{x \in \mathbb{R}^{2}:|x|<r\right\}$. The two-sphere $\left\{x \in \mathbb{R}^{3}:|x|=1\right\}$ is denoted by $S^{2}$.

The one-dimensional Hausdorff measure is denoted by $\mathcal{H}^{1}$.

Acknowledgments. The author would like to thank Stefan Müller for very helpful discussions.

\footnotetext{
${ }^{1}$ An immersion $y: M \rightarrow \mathbb{R}^{3}$ is short with respect to the metric $g$ on $M$ if for every curve $\gamma:[0,1] \rightarrow M$, the length of $y \circ \gamma$ is shorter (measured with the Euclidean metric on $\mathbb{R}^{3}$ ) than $\gamma$ (measured with $g$ ).
} 


\section{Proof of Theorem 1}

As in [32, the proof of the energy scaling law rests on two observations. First, by the weak formulation of the Hessian determinant,

$$
\sum_{i=1}^{3} \operatorname{det} D^{2} y_{i}=\left(y_{, 1} \cdot y_{, 2}\right)_{, 12}-\frac{1}{2}\left(\left|y_{, 1}\right|^{2}\right)_{, 22}-\frac{1}{2}\left(\left|y_{, 2}\right|^{2}\right)_{, 11} \quad \text { for } y \in C^{2}\left(B_{1} ; \mathbb{R}^{3}\right),
$$

we get that the quantity $\sum_{i=1}^{3} \operatorname{det} D^{2} y_{i}$ is close to $\sum_{i=1}^{3} \operatorname{det} D^{2} y_{i}^{\Delta}=\pi \Delta^{2} \delta_{0}$ in $W^{-2,2}$, where $\delta_{0}$ denotes the distribution $f \mapsto f(0)$. The expression $\sum_{i=1}^{3} \operatorname{det} D^{2} y_{i}$ is best thought of as the "linearized Gauss curvature": For a metric of the form $g_{y}=\operatorname{Id}_{2 \times 2}+\varepsilon G$, the Gauss curvature is $K=\varepsilon \sum_{i=1}^{3} \operatorname{det} D^{2} y_{i}+O\left(\varepsilon^{2}\right)$. Second, the following Sobolev/isoperimetric inequality translates estimates for integrals of the Hessian determinant into lower bounds for boundary integrals of the tangential part of the second derivative:

Lemma 1. For $v \in C^{2}\left(\overline{B_{1}}\right)$ and $0 \leq r \leq 1$,

$$
\int_{\partial B_{r}}\left|D^{2} v\right| \mathrm{d} \mathcal{H}^{1} \geq\left(4 \pi\left|\int_{B_{r}} \operatorname{det} D^{2} v \mathrm{~d} x\right|\right)^{1 / 2} .
$$

This inequality has been used in the literature in a number of places, see e.g. [27]. The proof of the statement above (including the sharp constant) can be found in [32].

The main observation that allows for an improvement of the lower bound from [32] is that we may get a lower bound for the quantity on the left hand side in (9) from the smallness of the membrane energy directly by integrating a suitable test function against the membrane term $g_{y}-g_{\Delta}$. In [32, we obtained such an estimate by interpolation instead, which also uses the control over the bending energy. This is unnecessary, and gives slightly worse estimates.

The following calculation indicates how to use the smallness of the membrane term to obtain estimates on integrals of the linearized curvature. Let $\Phi \in L^{1}\left(B_{1}\right)$ be such that $D^{2} \Phi$ is a vector-valued Radon measure with support in $B_{1}$. Then we have for all $y \in C^{2}\left(B_{1} ; \mathbb{R}^{3}\right)$ :

$$
\begin{aligned}
\int_{B_{1}}\left(\sum_{i=1}^{3} \operatorname{det}\right. & \left.D^{2} y_{i}(x)-\pi \Delta^{2} \delta_{0}\right) \Phi(x) \mathrm{d} \mathcal{L}^{2} \\
= & \int_{B_{1}}\left(\left(y_{, 1} \cdot y_{, 2}-y_{, 1}^{\Delta} \cdot y_{, 2}^{\Delta}\right) \Phi_{, 12}\right. \\
& \left.\quad-\frac{1}{2}\left(\left|y_{, 1}\right|^{2}-\left|y_{, 1}^{\Delta}\right|^{2}\right) \Phi_{, 22}-\frac{1}{2}\left(\left|y_{, 2}\right|^{2}-\left|y_{, 2}^{\Delta}\right|^{2}\right) \Phi_{, 11}\right) \mathrm{d} \mathcal{L}^{2} \\
= & -\frac{1}{2} \int_{B_{1}}\left(g_{y}-g_{\Delta}\right): \operatorname{cof} D^{2} \Phi \mathrm{d} \mathcal{L}^{2} .
\end{aligned}
$$

Here,

$$
\operatorname{cof} D^{2} \Phi=\left(\begin{array}{cc}
\Phi_{, 22} & -\Phi_{, 12} \\
-\Phi_{, 21} & \Phi_{, 11}
\end{array}\right)
$$

denotes the cofactor matrix of $D^{2} \Phi$. Note that cof is linear on two by two matrices, and hence cof $D^{2} \Phi$ is a well defined Radon measure under our assumptions. After these preliminary remarks, we construct the upper bound in the statement of Theorem 1. It is obtained by a simple mollification of $y^{\Delta}$ on a ball of size $h$ centered at the origin. 
Lemma 2. We have

$$
\inf _{y \in W^{2,2}\left(B_{1} ; \mathbb{R}^{3}\right)} I_{h, \Delta}(y) \leq 2 \pi \Delta^{2} h^{2}\left(\log \frac{1}{h}+C\right),
$$

where $C=C(\Delta)$ does not depend on $h$.

Proof. This is the same upper bound construction as in [32] (see Lemma 2 in that reference), and we will be brief. We choose $\eta \in C^{\infty}([0, \infty))$ with $\eta=0$ on $[0,1 / 2], \eta=1$ on $[1, \infty)$, and $\left|\eta^{\prime}\right| \leq C,\left|\eta^{\prime \prime}\right| \leq C$. We set

$$
y_{h}(x)=\eta(|x| / h) y^{\Delta}(x) .
$$

One easily shows

$$
\begin{aligned}
& \left|g_{y_{h}}-g_{\Delta}\right| \leq C \quad \text { and } \quad\left|D^{2} y_{h}\right| \leq C h^{-1} \quad \text { on } B_{h}, \\
& g_{y_{h}}-g_{\Delta}=0 \quad \text { and } \quad\left|D^{2} y_{h}(x)\right|=\Delta /|x| \quad \text { on } B_{1} \backslash B_{h} \text {. }
\end{aligned}
$$

This implies

$$
\begin{aligned}
\int_{B_{1}}\left|g_{y_{h}}-g_{\Delta}\right|^{2} \mathrm{~d} \mathcal{L}^{2} & \leq \int_{B_{h}} C \mathrm{~d} \mathcal{L}^{2} \\
& \leq C h^{2}, \\
\int_{B_{1}}\left|D^{2} y_{h}\right|^{2} \mathrm{~d} \mathcal{L}^{2} & \leq \int_{B_{1} \backslash B_{h}} \frac{\Delta^{2}}{|x|^{2}} \mathrm{~d} \mathcal{L}^{2}+\int_{B_{h}} \frac{C}{h^{2}} \mathrm{~d} \mathcal{L}^{2} \\
& =2 \pi \Delta^{2} \int_{h}^{1} \frac{\mathrm{d} r}{r}+C \\
& =2 \pi \Delta^{2} \log \frac{1}{h}+C .
\end{aligned}
$$

This implies the claim of the lemma.

Proof of Theorem 1. The upper bound is proved by Lemma 2, hence we may choose $C_{2}$ to be the constant from that lemma. Now it suffices to show the following: There exist $C_{1}, C_{3}$ such that if $y \in W^{2,2}\left(B_{1} ; \mathbb{R}^{3}\right)$ satisfies (4), then also the lower bound in (3) and (5), (6) hold true.

Let $y \in W^{2,2}\left(B_{1} ; \mathbb{R}^{3}\right)$ satisfy (4). By density of $C^{2}$ in $W^{2,2}$, we may assume $y \in C^{2}\left(B_{1} ; \mathbb{R}^{3}\right)$ for a proof of the remaining statements. Let $0<r<1$. Using Lemma 1, we have for $i=1,2,3$ :

$$
\frac{1}{2 \pi} \int_{\partial B_{r}}\left|D^{2} y_{i}\right| \mathrm{d} \mathcal{H}^{1} \geq\left(\frac{1}{\pi}\left|\int_{B_{r}} \operatorname{det} D^{2} y_{i} \mathrm{~d} \mathcal{L}^{2}\right|\right)^{1 / 2} .
$$

Applying Jensen's inequality, we get

$$
\frac{1}{2 \pi r} \int_{\partial B_{r}}\left|D^{2} y_{i}\right|^{2} \mathrm{~d} \mathcal{H}^{1} \geq\left(\frac{1}{2 \pi r} \int_{\partial B_{r}}\left|D^{2} y_{i}\right| \mathrm{d} \mathcal{H}^{1}\right)^{2} .
$$

Combining these two estimates, we obtain

$$
\int_{\partial B_{r}}\left|D^{2} y_{i}\right|^{2} \mathrm{~d} \mathcal{H}^{1} \geq \frac{2}{r}\left|\int_{B_{r}} \operatorname{det} D^{2} y_{i} \mathrm{~d} \mathcal{L}^{2}\right| .
$$

By the triangle inequality, 


$$
\int_{\partial B_{r}}\left|D^{2} y\right|^{2} \mathrm{~d} \mathcal{H}^{1} \geq \frac{2}{r}\left|\int_{B_{r}} \sum_{i} \operatorname{det} D^{2} y_{i} \mathrm{~d} \mathcal{L}^{2}\right| .
$$

Now choose $h_{0}=h_{0}(y) \in[h, 2 h]$ such that

$$
\int_{\partial B_{h_{0}}}\left|g_{y}-g_{\Delta}\right|^{2} \mathrm{~d} \mathcal{H}^{1} \leq C h^{-1} \int_{B_{1}}\left|g_{y}-g_{\Delta}\right|^{2} \mathrm{~d} \mathcal{L}^{2} .
$$

Choosing $h_{0}<R<1$ and integrating (11) over the range $r \in\left[h_{0}, R\right]$, we get

$$
\begin{aligned}
\int_{B_{R} \backslash B_{h_{0}}}\left|D^{2} y\right|^{2} \mathrm{~d} \mathcal{L}^{2} \\
\geq 2\left|\int_{h_{0}}^{R} \frac{1}{r}\left(\int_{B_{r}} \operatorname{det} D^{2} y_{i} \mathrm{~d} \mathcal{L}^{2}\right) \mathrm{d} r\right| \\
\geq 2 \int_{h_{0}}^{R} \frac{\pi \Delta^{2}}{r} \mathrm{~d} r \\
\quad-2\left|\int_{h_{0}}^{R} \mathrm{~d} r \int_{B_{1}} \mathrm{~d} x \frac{1}{r} \chi_{B_{r}}(x)\left(\pi \Delta^{2} \delta_{0}(x)-\sum_{i} \operatorname{det} D^{2} y_{i}(x)\right)\right| \\
=2 \pi \Delta^{2} \log \frac{R}{h_{0}}-2\left|\int_{B_{1}} \mathrm{~d} x \Phi(x)\left(\pi \Delta^{2} \delta_{0}(x)-\sum_{i} \operatorname{det} D^{2} y_{i}(x)\right)\right|,
\end{aligned}
$$

where we have used Fubini's Theorem to change the order of integration, and have defined the test function

$$
\Phi(x):=\int_{h_{0}}^{R} \frac{1}{r} \chi_{B_{r}}(x) \mathrm{d} r= \begin{cases}\log \frac{R}{h_{0}} & \text { if }|x| \leq h_{0} \\ \log \frac{R}{|x|} & \text { if } h_{0}<|x| \leq R \\ 0 & \text { else. }\end{cases}
$$

Now we set

$$
\begin{aligned}
A(R) & :=\int_{B_{1}}\left(\sum_{i} \operatorname{det} D^{2} y_{i}-\pi \Delta^{2} \delta_{0}\right) \Phi(x) \mathrm{d} \mathcal{L}^{2}(x) \\
& =-\frac{1}{2} \int_{B_{1}}\left(g_{y}-g_{\Delta}\right): \operatorname{cof} D^{2} \Phi \mathrm{d} \mathcal{L}^{2}(x),
\end{aligned}
$$

where we have used 10 in the second line. An explicit computation yields

$$
\begin{aligned}
D \Phi(x)= & -\frac{x}{|x|^{2}} \chi_{B_{R} \backslash B_{h_{0}}}(x) \\
D^{2} \Phi(x)= & \left(-\operatorname{Id}_{2 \times 2}+2 \hat{x} \otimes \hat{x}\right)|x|^{-2} \chi_{B_{R} \backslash B_{h_{0}}}(x) \\
& +|x|^{-1} \hat{x} \otimes \hat{x}\left(\mathcal { H } ^ { 1 } \left\llcorner\partial B_{R}-\mathcal{H}^{1}\left\llcorner\partial B_{h_{0}}\right) .\right.\right.
\end{aligned}
$$

Inserting these computations in (14), we have 


$$
\begin{aligned}
|A(R)| \leq & \int_{B_{R} \backslash B_{h_{0}}} \frac{\left|g_{y}-g_{\Delta}\right|}{|x|^{2}} \mathrm{~d} \mathcal{L}^{2}+\frac{1}{2 R} \int_{\partial B_{R}}\left|g_{y}-g_{\Delta}\right| \mathrm{d} \mathcal{H}^{1} \\
& +\frac{1}{2 h_{0}} \int_{\partial B_{h_{0}}}\left|g_{y}-g_{\Delta}\right| \mathrm{d} \mathcal{H}^{1}
\end{aligned}
$$

By Cauchy-Schwarz,

$$
\begin{aligned}
\int_{B_{R} \backslash B_{h_{0}}} \frac{\left|g_{y}-g_{\Delta}\right|}{|x|^{2}} \mathrm{~d} \mathcal{L}^{2} & \leq\left(\int_{B_{R} \backslash B_{h_{0}}}\left|g_{y}-g_{\Delta}\right|^{2} \mathrm{~d} \mathcal{L}^{2}\right)^{1 / 2}\left(\int_{B_{R} \backslash B_{h_{0}}}|x|^{-4} \mathrm{~d} \mathcal{L}^{2}\right)^{1 / 2} \\
& \leq\left(\int_{B_{R} \backslash B_{h_{0}}}\left|g_{y}-g_{\Delta}\right|^{2} \mathrm{~d} \mathcal{L}^{2}\right)^{1 / 2} \sqrt{2 \pi} h_{0}^{-1} \\
\int_{\partial B_{R}}\left|g_{y}-g_{\Delta}\right| \mathrm{d} \mathcal{H}^{1} & \leq C \sqrt{R}\left(\int_{\partial B_{R}}\left|g_{y}-g_{\Delta}\right|^{2} \mathrm{~d} \mathcal{H}^{1}\right)^{1 / 2} \\
\int_{\partial B_{h_{0}}}\left|g_{y}-g_{\Delta}\right| \mathrm{d} \mathcal{H}^{1} & \leq C \sqrt{h_{0}}\left(\int_{\partial B_{h_{0}}}\left|g_{y}-g_{\Delta}\right|^{2} \mathrm{~d} \mathcal{H}^{1}\right)^{1 / 2} .
\end{aligned}
$$

Now choose $R_{0} \in[R-h, R]$ such that

$$
\int_{\partial B_{R_{0}}}\left|g_{y}-g_{\Delta}\right|^{2} \mathrm{~d} \mathcal{H}^{1} \leq C h^{-1} \int_{B_{1}}\left|g_{y}-g_{\Delta}\right|^{2} \mathrm{~d} \mathcal{L}^{2} .
$$

Together with (12) and (16), 15 becomes

$$
\left|A\left(R_{0}\right)\right| \leq C \frac{E_{\mathrm{m}}(y)^{1 / 2}}{h_{0}},
$$

where $E_{\mathrm{m}}(y)$ is the membrane energy,

$$
E_{\mathrm{m}}(y):=\int_{B_{1}}\left|g_{y}-g_{\Delta}\right|^{2} \mathrm{~d} \mathcal{L}^{2} .
$$

The lower bound for the bending energy (13) becomes

$$
\int_{B_{R_{0}} \backslash B_{h_{0}}}\left|D^{2} y\right|^{2} \mathrm{~d} \mathcal{L}^{2} \geq 2 \pi \Delta^{2} \log \frac{R_{0}}{h_{0}}-C \frac{E_{\mathrm{m}}(y)^{1 / 2}}{h_{0}} .
$$

We use (17) with $R \uparrow 1$ to estimate the membrane energy by

$$
\begin{aligned}
E_{\mathrm{m}}(y) & \leq 2 \pi \Delta^{2} h^{2}\left(\log \frac{1}{h}+C_{2}\right)-2 \pi \Delta^{2} h^{2} \log \frac{1}{h_{0}}+C h^{2} \frac{E_{\mathrm{m}}(y)^{1 / 2}}{h_{0}} \\
& \leq C\left(h^{2}+h E_{\mathrm{m}}(y)^{1 / 2}\right) .
\end{aligned}
$$

Using Young's inequality $a b \leq \frac{1}{2}\left((\varepsilon a)^{2}+(b / \varepsilon)^{2}\right)$, with $\varepsilon=C^{-1}$, we have

$$
C h E_{\mathrm{m}}(y)^{1 / 2} \leq \frac{1}{2} E_{\mathrm{m}}(y)+C h^{2},
$$


and inserting this in (18), we get

$$
E_{\mathrm{m}}(y) \leq C h^{2},
$$

which proves (6). Furthermore, inserting this in (17), we have

$$
\int_{B_{R_{0}} \backslash B_{h_{0}}}\left|D^{2} y\right|^{2} \mathrm{~d} \mathcal{L}^{2} \geq 2 \pi \Delta^{2} \log \frac{R_{0}}{h}-C .
$$

Sending $R \rightarrow 1$, this proves the lower bound in (3). Furthermore,

$$
\begin{aligned}
\int_{B_{1} \backslash B_{R}}\left|D^{2} y\right|^{2} \mathrm{~d} \mathcal{L}^{2} & \leq h^{-2}\left(I_{h, \Delta}(y)-E_{\mathrm{m}}(y)\right)-\int_{h_{0}}^{R_{0}}\left|D^{2} y\right|^{2} \mathrm{~d} \mathcal{L}^{2} \\
& \leq 2 \pi \Delta^{2}\left(\log 1 / h+C_{2}\right)-2 \pi \Delta^{2} \log \frac{R_{0}}{h} \\
& \leq 2 \pi \Delta^{2} \log \frac{1}{R}+C
\end{aligned}
$$

which proves (5). This completes the proof of the theorem.

\section{Proof of Theorem 2}

3.1. Isometric immersions of a singular cone. The plan of the proof is as follows: The crucial inequality (5) shows that on a fixed annulus $B_{1} \backslash B_{R}$, the $W^{2,2}$ norm of a sequence of deformations $y_{h}$ satisfying $I_{h, \Delta}\left(y_{h}\right) \leq 2 \pi \Delta^{2} h^{2}(\log 1 / h+C)$ is bounded as $h \rightarrow 0$. One gets weak convergence in $W^{2,2}$ to a limit deformation that is an isometric immersion with respect to $g_{\Delta}$ (since the membrane energy of the limit function vanishes by $E_{\mathrm{m}}\left(y_{h}\right) \leq C h^{2} \rightarrow 0$ ). We may apply the results on $W^{2,2}$ isometric immersions from [15, 33] to the limit, which means that the limit deformation is developable. Using our energy estimates, we can show that in fact, it must be identical to the singular cone $y^{\Delta}$ up to a Euclidean motion.

The fact that flat surfaces are locally developable is a classical result from Differential Geometry of surfaces. For functions in $W^{2,2}$, this statement has been proved in [15, 16, 33]:

Theorem 3 (Theorem 2 in [15]). Let $\Omega \subset \mathbb{R}^{2}$ with Lipschitz boundary. Let $y \in W^{2,2}\left(\Omega ; \mathbb{R}^{3}\right)$ with $D y^{T} D y=\operatorname{Id}_{2 \times 2}$. Then $y \in C^{1}(\Omega)$ and there exists a set $\mathbf{L}_{y}$ of mutually disjoint closed line segments in $\bar{\Omega}$ with endpoints on $\partial \Omega$ with the following property: For every $x \in \Omega$, either $D^{2} y=0$ in a neighborhood of $x$, or there exists $L \in \mathbf{L}_{y}$ with $x \in L$ and $D y$ is constant on $L$.

We will need a variant of this theorem for functions whose domain is a singular cone.

To be able to use Theorem 3 , we are going to consider the cone in a flat reference configuration. Let arccos $:[-1,1] \rightarrow[0, \pi]$ denote the inverse of $\cos :[0, \pi] \rightarrow[-1,1]$. Define

$$
B_{1, \Delta}:=\left\{x=\left(x_{1}, x_{2}\right) \in B_{1} \backslash\{0\}: 0 \leq \arccos \frac{x_{1}}{|x|}<\sqrt{1-\Delta^{2}} \pi\right\} .
$$

Let $\mathbb{R}_{-}:=\left\{\left(x_{1}, 0\right): x_{1} \leq 0\right\}$, and let $\varphi: \mathbb{R}^{2} \backslash \mathbb{R}_{-} \rightarrow \mathbb{R}$ be the angular coordinate satisfying $x=|x|(\cos \varphi(x), \sin \varphi(x))$ with values in $(-\pi, \pi)$. We define the map $\iota \equiv \iota_{\Delta}: \mathbb{R}^{2} \backslash \mathbb{R}_{-} \rightarrow B_{1}$ by

$$
\iota(x)=\left(|x| \cos \frac{\varphi(x)}{\sqrt{1-\Delta^{2}}},|x| \sin \frac{\varphi(x)}{\sqrt{1-\Delta^{2}}}\right) .
$$



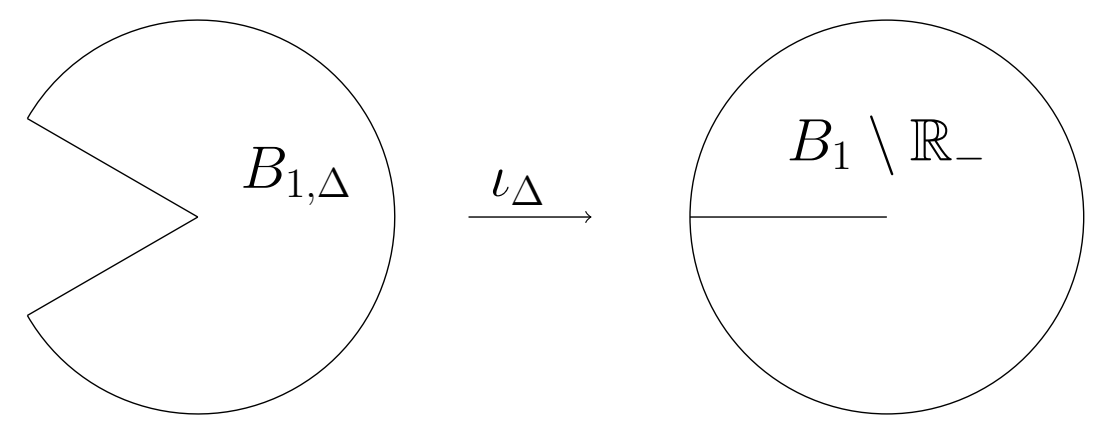

Figure 1 . The domain $B_{1, \Delta}$ and the map $\iota_{\Delta}: B_{1, \Delta} \rightarrow B_{1} \backslash \mathbb{R}_{-}$.

For a sketch of $B_{1, \Delta}$ and $\iota_{\Delta}$, see Figure 1

On $\iota\left(B_{1, \Delta}\right)=B_{1} \backslash \mathbb{R}_{-}, \iota$ has a well defined inverse, that we denote by

$$
j: B_{1} \backslash \mathbb{R}_{-} \rightarrow B_{1, \Delta} .
$$

Furthermore, let $\phi_{\Delta}:=\left(1-\sqrt{1-\Delta^{2}}\right) 2 \pi$ and let the rotation $S_{\Delta} \in S O(2)$ be defined by

$$
S_{\Delta}=\left(\begin{array}{cc}
\cos \phi_{\Delta} & -\sin \phi_{\Delta} \\
\sin \phi_{\Delta} & \cos \phi_{\Delta}
\end{array}\right) \text {. }
$$

Finally, let

$$
\partial_{\Delta}:=\partial B_{1, \Delta} \backslash\left(\partial B_{1} \cup\{0\}\right)
$$

Note that $\partial_{\Delta}$ has two connected components, one contained in the upper half plane and one in the lower half plane. We will denote them by $\partial_{\Delta}^{+}$and $\partial_{\Delta}^{-}$respectively, see Figure 2 . The rotation matrix $S_{\Delta}$ has been chosen such that $S_{\Delta} \partial_{\Delta}^{+}=\partial_{\Delta}^{-}$.

We define

$$
\begin{aligned}
W_{\text {iso }}^{2,2}\left(B_{1, \Delta}\right):= & \left\{Y \in W_{\text {loc }}^{2,2}\left(B_{1, \Delta} ; \mathbb{R}^{3}\right): g_{Y}=\operatorname{Id}_{2 \times 2},\right. \\
& \left.Y\left(S_{\Delta} x\right)=Y(x) \text { and } D Y\left(S_{\Delta} x\right)=D Y(x) S_{\Delta} \text { for every } x \in \partial_{\Delta}^{+} \cdot\right\}
\end{aligned}
$$

This definition is chosen such that if $y \in W_{\text {loc }}^{2,2}\left(B_{1} \backslash\{0\} ; \mathbb{R}^{3}\right)$ with $D y^{T} D y=g_{\Delta}$, then $y \circ \iota \in W_{\text {iso }}^{2,2}\left(B_{1, \Delta}\right)$.

To $Y \in W_{\text {iso }}^{2,2}\left(B_{1, \Delta}\right)$, we may apply Theorem 3 (with $\Omega=B_{1, \Delta} \backslash B_{\rho}$ ) to obtain a set $\mathbf{L}_{Y}$ of line segments with the properties stated there. In fact, by sending $\rho \rightarrow 0$, we get a set of (relatively) closed mutually disjoint line segments in $\overline{B_{1, \Delta}} \backslash\{0\}$. If a line segment has only one endpoint in $\overline{B_{1, \Delta}} \backslash\{0\}$, then we say by slight abuse of terminology that one of its endpoints is the origin.

Next, we are going to define an "adjoint" line segment $L^{\text {ad }}$ to any $L \in \mathbf{L}_{Y}$ with an endpoint $x \in \partial_{\Delta}$. Note that for such $L$, there exists $v \in \partial B_{1}$ and $q>0$ such that

$$
L=\{x+t v: t \in[0, q]\} .
$$


First let us assume $x \in \partial_{\Delta}^{+}$. By the definition of $W_{\text {iso }}^{2,2}\left(B_{1, \Delta}\right)$ in $(19)$, we have that $x^{\prime}:=S_{\Delta} x \in \partial_{\Delta}^{-}$, and $D Y\left(x^{\prime}\right)=D Y(x) S_{\Delta}$. Moreover, there has to exist $L^{\text {ad }} \in \mathbf{L}_{y}$ with $x^{\prime} \in L^{\text {ad }}$, and $L^{\text {ad }}=\left\{x^{\prime}+t S_{\Delta} v: t \in \mathbb{R}\right\} \cap \overline{B_{1, \Delta}}$. This defines $L^{\text {ad }}$ for $x \in \partial_{\Delta}^{+}$; for $x \in \partial_{\Delta}^{-}$, we define it analogously, replacing $S_{\Delta}$ by $S_{\Delta}^{-1}$. For a sketch of the construction, see Figure 2 .

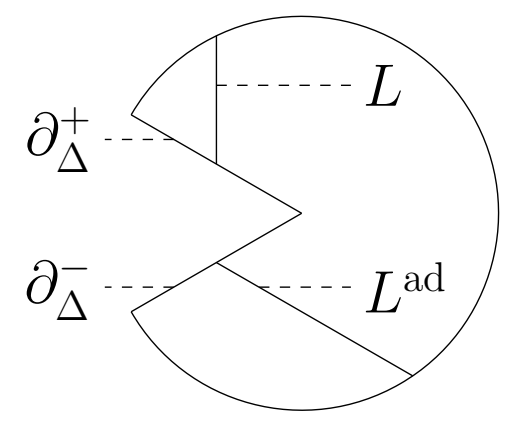

Figure 2. The subsets $\partial_{\Delta}^{+}, \partial_{\Delta}^{-}$of the boundary and adjoint line segments $L, L^{\text {ad }}$.

From now on, the line segments in $\mathbf{L}_{Y}$ for which one of the endpoints is 0 will be called "good", and line segments in the complement of the set of good line segments will be called "bad". The sets of good and bad line segments will be denoted by $\mathbf{L}_{Y}^{(g)}, \mathbf{L}_{Y}^{(b)}$ respectively. For any bad line segment, we can lower the elastic energy by "flattening" the deformation $Y$ on one side of the line segment. This is the idea behind the following lemma. For a sketch of this operation, see Figure 3 .
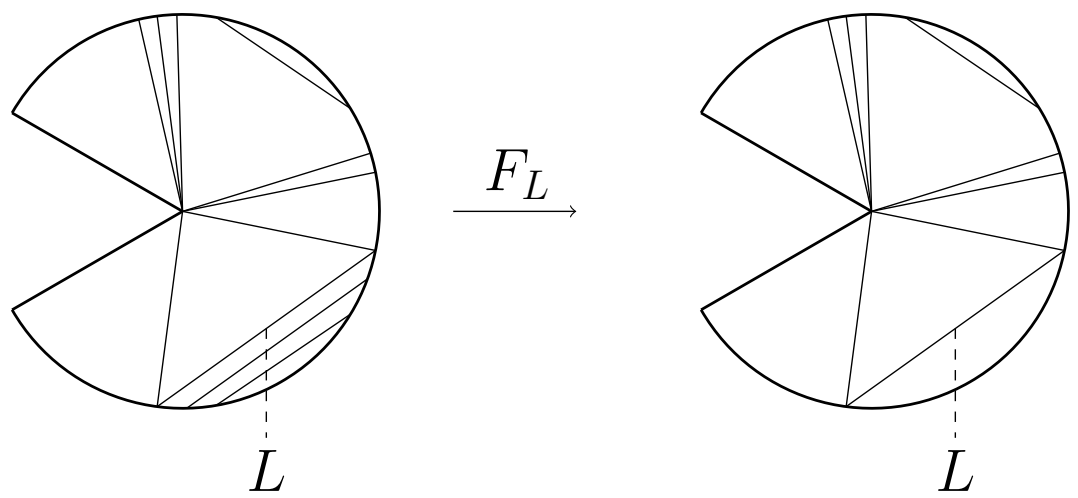

Figure 3. In the left panel, we have the segments that belong to $\mathbf{L}_{Y}$, and $L \in \mathbf{L}_{Y}$ is a bad line segment. We can flatten the deformation $Y$ on the side of $L$ whose closure does not contain the origin, and obtain a deformation $F_{L}(Y)$, such that $\mathbf{L}_{F_{L}(Y)}$ consists of those line segments in $\mathbf{L}_{Y}$ that are on the same side of $L$ as the origin, see the right panel.

Lemma 3. For every $Y \in W_{\text {iso }}^{2,2}\left(B_{1, \Delta}\right)$, there exists $Y_{\infty} \in W_{\text {iso }}^{2,2}\left(B_{1, \Delta}\right)$ with the following properties: 
(i) $\mathbf{L}_{Y_{\infty}}^{(b)}=\emptyset$ and $\mathbf{L}_{Y_{\infty}}^{(g)}=\mathbf{L}_{Y}^{(g)}$

(ii) For $0<\rho<1$, we have

$$
\begin{aligned}
\int_{B_{1, \Delta} \backslash B_{\rho}} \mid D^{2} Y_{\infty} & :\left.\left((D \iota)^{-1} \otimes(D \iota)^{-1}\right)\right|^{2} \mathrm{~d} \mathcal{L}^{2} \\
& \leq \int_{B_{1, \Delta} \backslash B_{\rho}}\left|D^{2} Y:\left((D \iota)^{-1} \otimes(D \iota)^{-1}\right)\right|^{2} \mathrm{~d} \mathcal{L}^{2},
\end{aligned}
$$

with equality for all $0<\rho<1$ if and only if $Y=Y_{\infty}$.

Proof. For any $L \in \mathbf{L}_{Y}^{(b)}$, we may define a modified map $F_{L}(Y) \in W_{\text {iso }}^{2,2}\left(B_{1, \Delta}\right)$ as follows. On $L$, we have $Y=A_{L} x+b_{L}$ for some $A_{L} \in \mathbb{R}^{3 \times 2}$ and $b_{L} \in \mathbb{R}^{3}$. We note that $B_{1, \Delta} \backslash L$ has exactly two connected components. Let $E_{L}$ denote the connected component whose closure does not contain the origin. First let us assume that none of the endpoints of $L$ is in $\partial_{\Delta}$. Then we define $F_{L}(Y) \in W_{\text {iso }}^{2,2}\left(B_{1, \Delta}\right)$ by

$$
F_{L}(Y)(x)= \begin{cases}A_{L} x+b_{L} & \text { if } x \in E_{L} \\ Y(x) & \text { else. }\end{cases}
$$

If one of the endpoints of $L$ is in $\partial_{\Delta}$, then we set

$$
F_{L}(Y)(x)= \begin{cases}A_{L}+b_{L} & \text { if } x \in E_{L} \\ A_{L^{\mathrm{ad}}} x+b_{L^{\mathrm{ad}}} & \text { if } x \in E_{L^{\mathrm{ad}}} \\ Y(x) & \text { else. }\end{cases}
$$

Note that this definition indeed satisfies $F_{L}(Y) \in W_{\text {iso }}^{2,2}\left(B_{1, \Delta}\right)$. Obviously, we have $D^{2}\left(F_{L}(Y)\right)=$ 0 on $E_{L}$ (and on $\left.E_{L^{\text {ad }}}\right)$ and hence, for all $0<\rho<1$, we have

$$
\int_{B_{1, \Delta} \backslash B_{\rho}}\left|D^{2} F_{L}(Y)(D \iota)^{-1}\right|^{2} \mathrm{~d} \mathcal{L}^{2} \leq \int_{B_{1, \Delta} \backslash B_{\rho}}\left|D^{2} Y(D \iota)^{-1}\right|^{2} \mathrm{~d} \mathcal{L}^{2}
$$

We must distinguish two cases in 22 : If $\mathbf{L}_{F_{L}(Y)} \subsetneq \mathbf{L}_{Y}$, then $F_{L}(Y) \neq Y$ and we must have $\left|D^{2} Y\right|>0$ on a subset of positive measure of $E_{L}$. Hence, inequality must hold in (22) for some $\rho$, since we have

$$
\sqrt{1-\Delta^{2}} \operatorname{Id}_{2 \times 2} \leq(D \iota)^{-1} \leq \operatorname{Id}_{2 \times 2}
$$

in the sense of positive definite matrices. Equality in 22 only holds in the case $F_{L}(Y)=$ $Y$.

On $\mathbf{L}_{Y}^{(b)}$, we may define an order relation by $L<L^{\prime}$ if $E_{L} \subsetneq E_{L^{\prime}}$. Since bad line segments are mutually disjoint, we have that either $L<L^{\prime}, L>L^{\prime}$ or $E_{L} \cap E_{L^{\prime}}=\emptyset$. Hence, there exists an at most countable sequence $L_{1}, L_{2}, \ldots$ of maximal bad line segments. If for two maximal line segments $L, L^{\prime}$ we have $L^{\prime}=L^{\text {ad }}$ then we exclude exactly one of them from that sequence. Now we define a sequence $Y_{k} \in W_{\text {iso }}^{2,2}\left(B_{1, \Delta}\right)$ by

$$
Y_{k}=F_{L_{k}} \circ \cdots \circ F_{L_{1}}(Y) \text {. }
$$

By (22) and (23), $D^{2} Y_{k}$ is bounded in $L^{2}$. Thus the sequence converges weakly in $W^{2,2}\left(B_{1, \Delta} \backslash B_{\rho} ; \mathbb{R}^{3}\right)$ for every $0<\rho<1$ to a limit $Y_{\infty} \in W_{\text {iso }}^{2,2}\left(B_{1, \Delta}\right)$ such that $\mathbf{L}_{Y_{\infty}}$ does not contain any bad line segments, and $\mathbf{L}_{Y_{\infty}}^{(g)}=\mathbf{L}_{Y}^{(g)}$. The claim 20) follows from 22 and the comment after that equation. This proves the lemma. 
Remark 1. Letting $Y, Y_{\infty}$ as in Lemma 3, we have

$$
\begin{aligned}
Y_{\infty} \circ j & \in W_{\text {loc }}^{2,2}\left(B_{1} \backslash\{0\} ; \mathbb{R}^{3}\right) \\
g_{Y_{\infty} \circ j} & =g_{\Delta} .
\end{aligned}
$$

Furthermore,

$$
\int_{B_{1} \backslash B_{\rho}}\left|D^{2}\left(Y_{\infty} \circ j\right)\right|^{2} \mathrm{~d} \mathcal{L}^{2} \leq \int_{B_{1} \backslash B_{\rho}}\left|D^{2}(Y \circ j)\right|^{2} \mathrm{~d} \mathcal{L}^{2} \quad \text { for every } 0<\rho<1 .
$$

Proof. The first two statements follows immediately from $Y_{\infty} \in W_{\text {iso }}^{2,2}\left(B_{1, \Delta}\right)$, and only the inequality requires a proof. Let $\nu=Y_{, 1} \wedge Y_{, 2} /\left|Y_{, 1} \wedge Y_{, 2}\right|$ be the unit normal. By $D Y^{T} D Y=\operatorname{Id}_{2 \times 2}$, we have $D^{2} Y \perp D Y$. Hence

$$
\begin{aligned}
\left|D^{2}(Y \circ j)\right|^{2} & =\left|D^{2} Y:(D j \otimes D j)+D Y D^{2} j\right|^{2} \\
& =\left|D^{2} Y:(D j \otimes D j)\right|^{2}+\left|D Y D^{2} j\right|^{2} \\
& =\left|D^{2} Y:(D j \otimes D j)\right|^{2}+\left|D^{2} j\right|^{2},
\end{aligned}
$$

where we used $D Y \in O(2,3)$ in the last equality. Now the claim of the remark follows from 20 and a change of variables in the integrals.

\subsection{Proof of Theorem 2 .}

Proof of Theorem 2. To prove the convergence (7), it is enough to prove that for any subsequence of $y_{h}$, there exists a further subsequence such that the convergence (7) holds. Hence, we assume that we start with an arbitrary subsequence, and may take further subsequences at will.

Given $0<R<1$, we may assume that $h \ll R$. Choose $R_{0}(h) \in[R-h, R]$ such that

$$
\int_{\partial B_{R_{0}(h)}}\left|g_{y_{h}}-g_{\Delta}\right|^{2} \mathrm{~d} \mathcal{H}^{1} \leq h^{-1} \int_{B_{1}}\left|g_{y_{h}}-g_{\Delta}\right|^{2} \mathrm{~d} \mathcal{L}^{2}
$$

By Theorem 1, we have

$$
\begin{aligned}
\int_{B_{1} \backslash B_{R}}\left|D^{2} y_{h}\right| \mathrm{d} \mathcal{L}^{2} & \leq \int_{B_{1} \backslash B_{R_{0}}}\left|D^{2} y_{h}\right| \mathrm{d} \mathcal{L}^{2} \\
& \leq 2 \pi \Delta^{2} \log \frac{1}{R}+C,
\end{aligned}
$$

where $C$ does neither depend on $h$ nor on $R$. This proves the boundedness of $y_{h}$ in $W^{2,2}\left(B_{1} \backslash B_{R} ; \mathbb{R}^{3}\right)$ and implies that there exists $\hat{y}_{R} \in W^{2,2}\left(B_{1} \backslash B_{R} ; \mathbb{R}^{3}\right)$ such that (for a subsequence)

$$
y_{h} \rightarrow \hat{y}_{R} \quad \text { in } W^{2,2}\left(B_{1} \backslash B_{R} ; \mathbb{R}^{3}\right) .
$$

After taking a suitable diagonal sequence for $R=\frac{1}{j}, j=2,3, \ldots$, we may assume that $\hat{y}_{R} \in W_{\text {loc. }}^{2,2}\left(B_{1} \backslash\{0\} ; \mathbb{R}^{3}\right)$ is independent of $R$. We denote this function by $y^{*}$. By Theorem 1. we have

$$
\int_{B_{1}}\left|g_{y^{*}}-g_{\Delta}\right| \mathrm{d} \mathcal{L}^{2}=0
$$


I.e., $y^{*}$ is an isometry with respect to $g_{\Delta}$.

By (26), we have

$$
\int_{B_{1} \backslash B_{R}}\left|D^{2} y^{*}\right|^{2} \mathrm{~d} \mathcal{L}^{2} \leq 2 \pi \Delta^{2} h^{2} \log \frac{1}{R}+C
$$

Let $Y: B_{1, \Delta} \rightarrow \mathbb{R}^{3}$ be defined by

$$
Y:=y^{*} \circ \iota
$$

Recalling the definitions from Section 3.1. we have $Y \in W_{\text {iso }}^{2,2}\left(B_{1, \Delta}\right)$. By an application of Lemma 3, we obtain $Y_{\infty} \in W_{\text {iso }}^{2,2}\left(B_{1, \Delta}\right)$ such that $D Y_{\infty}$ is constant on every line segment $(0, x)$ with $x \in \partial B_{1, \Delta} \cap \partial B_{1}$. Now we set $y^{\infty}:=Y_{\infty} \circ j$, and obtain that $D y^{\infty}$ is constant on every line segment $(0, x]$ with $x \in \partial B_{1}$. Hence there exists a curve $\gamma: \partial B_{1} \rightarrow S^{2}$ satisfying $\left|\gamma^{\prime}\right|=\sqrt{1-\Delta^{2}}$ such that

$$
y^{\infty}(x)=x \gamma(x /|x|)
$$

Using this expression, explicit computation yields

$$
\int_{\partial B_{\rho}}\left|D^{2} y^{\infty}\right|^{2} \mathrm{~d} \mathcal{H}^{1}=\frac{1}{\rho} \int_{\partial B_{1}}\left|D^{2} y^{\infty}\right|^{2} \mathrm{~d} \mathcal{H}^{1}
$$

By Remark 1 and (27), we have that for every $0<\rho<1$,

$$
\int_{B_{1} \backslash B_{\rho}}\left|D^{2} y^{\infty}\right|^{2} \mathrm{~d} \mathcal{L}^{2} \leq 2 \pi \Delta^{2} \log \frac{1}{\rho}+C
$$

Combining $(29)$ and $(30)$, we see that for every $0<\rho<1$, we have

$$
\int_{\partial B_{\rho}}\left|D^{2} y^{\infty}\right|^{2} \mathrm{~d} \mathcal{H}^{1} \leq \frac{2 \pi \Delta^{2}}{\rho}
$$

and the constant $C$ in $(30)$ is in fact 0 .

By $g_{y^{\infty}}=g_{\Delta}$, we have

$$
\sum_{i=1}^{3} \operatorname{det} D^{2} y_{i}^{\infty}=\pi \Delta^{2} \delta_{0}
$$

distributionally. We may now estimate using Lemma 1 for any $0<\rho<1$, 


$$
\begin{aligned}
\pi \Delta^{2} & =\int_{B_{\rho}} \sum_{i=1}^{3} \operatorname{det} D^{2} y_{i}^{\infty} \mathrm{d} \mathcal{L}^{2} \\
& \leq \sum_{i}\left|\int_{B_{\rho}} \operatorname{det} D^{2} y_{i}^{\infty} \mathrm{d} \mathcal{L}^{2}\right| \\
& \leq \frac{1}{4 \pi} \sum_{i}\left(\int_{\partial B_{\rho}}\left|D^{2} y_{i}^{\infty}(x) \cdot \hat{x}^{\perp}\right| \mathrm{d} \mathcal{H}^{1}(x)\right)^{2} \\
& \leq \frac{1}{4 \pi} \sum_{i} 2 \pi \rho\left(\int_{\partial B_{\rho}}\left|D^{2} y_{i}^{\infty}(x) \cdot \hat{x}^{\perp}\right|^{2} \mathrm{~d} \mathcal{H}^{1}(x)\right) \\
& \leq \frac{\rho}{2} \int_{\partial B_{\rho}}\left|D^{2} y^{\infty}(x) \cdot \hat{x}^{\perp}\right|^{2} \mathrm{~d} \mathcal{H}^{1}(x) \\
& \leq \pi \Delta^{2} .
\end{aligned}
$$

Here, to obtain the fourth from the third line, we have used Jensen's inequality. By this chain of estimates, all the inequalities must have been equalities, and we have

$$
\left(\int_{\partial B_{\rho}} \sum_{i}\left|D^{2} y_{i}^{\infty}(x) \cdot \hat{x}^{\perp}\right| \mathrm{d} \mathcal{H}^{1}(x)\right)^{2}=2 \pi \rho\left(\int_{\partial B_{\rho}}\left|D^{2} y_{i}^{\infty}(x) \cdot \hat{x}^{\perp}\right|^{2} \mathrm{~d} \mathcal{H}^{1}(x)\right)
$$

and thus

$$
\left|D^{2} y_{i}^{\infty}(x) \cdot \hat{x}^{\perp}\right|^{2}=\text { constant } \quad \text { for } x \in \partial B_{\rho}, i \in\{1,2,3\} .
$$

Additionally, (31) implies that

$$
\left|D^{2} y^{\infty}(x) \cdot \hat{x}^{\perp}\right|^{2}=\frac{\Delta^{2}}{\rho^{2}} \quad \text { for } x \in \partial B_{\rho} .
$$

By (28), we have $D^{2} y^{\infty}(x)=|x|^{-1}\left(\gamma+\gamma^{\prime \prime}\right) \otimes \hat{x}^{\perp} \otimes \hat{x}^{\perp}$. Combining this with (32), we get

$$
\left(\gamma+\gamma^{\prime \prime}\right) \cdot e_{i}=\text { constant on } \partial B_{1}
$$

for $i=1,2,3$. We write $c_{i}=\left(\gamma+\gamma^{\prime \prime}\right) \cdot e_{i}$, and have $D^{2} y_{i}^{\infty}(x)=\frac{c_{i}}{|x|} \hat{x}^{\perp} \otimes \hat{x}^{\perp}$, which implies

$$
y_{i}^{\infty}(x)=c_{i}|x|+a_{i} \cdot x+b_{i} \quad \text { for } i=1,2,3,
$$

for some $a_{i} \in \mathbb{R}^{2}, b_{i} \in \mathbb{R}$. By 32 we obtain

$$
\left|D^{2} y^{\infty}(x)\right|^{2}=\frac{\sum_{i} c_{i}^{2}}{|x|^{2}}=\frac{\Delta^{2}}{|x|^{2}}
$$

and thus $\sum_{i} c_{i}^{2}=\Delta^{2}$. By $g_{y^{\infty}}=g_{\Delta}$, we have

$$
\begin{aligned}
\operatorname{Id}_{2 \times 2}-\Delta^{2} \hat{x}^{\perp} \otimes \hat{x}^{\perp} & =(c \otimes \hat{x}+a)^{T}(c \otimes x+a) \\
& =|c|^{2} \hat{x} \otimes \hat{x}+(c \cdot a) \otimes \hat{x}+\hat{x} \otimes(c \cdot a)+a^{T} a .
\end{aligned}
$$

This yields

$$
\left(1-\Delta^{2}\right) \operatorname{Id}_{2 \times 2}=(c \cdot a) \otimes \hat{x}+\hat{x} \otimes(c \cdot a)+a^{T} a,
$$


which can only hold true for all $\hat{x} \in \partial B_{1}$ if $c \cdot a=0$ and $a^{T} a=\left(1-\Delta^{2}\right) \operatorname{Id}_{2 \times 2}$. This implies that

$$
R:=\left(\frac{a}{\sqrt{1-\Delta^{2}}}, \frac{c}{\Delta}\right) \in O(3)
$$

is an orthogonal matrix, and we have

$$
y^{\infty}(x)=R\left(\sqrt{1-\Delta^{2}} x+\Delta e_{3}|x|\right)+b .
$$

It remains to show that $y^{\infty}=y^{*}$. To see this, note that $y^{\infty} \circ \iota=Y_{\infty}$ satisfies

$$
\left\{(0, x]: x \in \partial B_{1, \Delta} \cap \partial B_{1}\right\}=\mathbf{L}_{Y_{\infty}}^{(g)}=\mathbf{L}_{Y}^{(g)},
$$

where the second equality holds by Lemma 3 . This implies that for every $x \in B_{1, \Delta}$ there exists an $L \in \mathbf{L}_{Y}^{(g)}$ with $x \in L$. This in turn implies that $\mathbf{L}_{Y}^{(b)}=\emptyset$ (since the line segments in $\mathbf{L}_{Y}$ are pairwise disjoint). By Lemma 3, the latter yields $Y=Y_{\infty}$. Composing with $j$ on both sides of this last equation, we obtain $y^{*}=y^{\infty}$. This completes the proof of the theorem.

\section{REFERENCES}

[1] P. Bella and R. V. Kohn. Metric-induced wrinkling of a thin elastic sheet. J. Nonlinear Sci., 24(6):1147-1176, 2014.

[2] P. Bella and R. V. Kohn. Wrinkles as the result of compressive stresses in an annular thin film. Comm. Pure Appl. Math., 67(5):693-747, 2014.

[3] M. Ben Amar and Y. Pomeau. Crumpled paper. Proc. Roy. Soc. London Ser. A, 453(1959):729-755, 1997.

[4] H. Ben Belgacem, S. Conti, A. DeSimone, and S. Müller. Energy scaling of compressed elastic films - three-dimensional elasticity and reduced theories. Arch. Ration. Mech. Anal., 164(1):1-37, 2002.

[5] J. Brandman, R. V. Kohn, and H.-M. Nguyen. Energy scaling laws for conically constrained thin elastic sheets. J. Elasticity, 113(2):251-264, 2013.

[6] E. Cerda, S. Chaieb, F. Melo, and L. Mahadevan. Conical dislocations in crumpling. Nature, 401:46-49, 1999.

[7] E. Cerda and L. Mahadevan. Conical surfaces and crescent singularities in crumpled sheets. Phys. Rev. Lett., 80:2358-2361, Mar 1998.

[8] E. Cerda and L. Mahadevan. Confined developable elastic surfaces: cylinders, cones and the elastica. Proceedings of the Royal Society A: Mathematical, Physical and Engineering Science, 461(2055):671-700, 2005.

[9] P. Constantin, W. E, and E. S. Titi. Onsager's conjecture on the energy conservation for solutions of euler's equation. Comm. Math. Phys., 165(1):207-209, 1994.

[10] S. Conti and F. Maggi. Confining thin elastic sheets and folding paper. Arch. Ration. Mech. Anal., 187(1):1-48, 2008.

[11] B. A. DiDonna and T. A. Witten. Anomalous strength of membranes with elastic ridges. Phys. Rev. Lett., 87:206105, Oct 2001.

[12] G. Friesecke, R. D. James, and S. Müller. A theorem on geometric rigidity and the derivation of nonlinear plate theory from three-dimensional elasticity. Comm. Pure Appl. Math., 55(11):1461-1506, 2002. 
[13] G. Friesecke, R. D. James, and S. Müller. A hierarchy of plate models derived from nonlinear elasticity by Gamma-convergence. Arch. Ration. Mech. Anal., 180(2):183$236,2006$.

[14] M. Gromov. Partial differential relations, volume 9 of Ergebnisse der Mathematik und ihrer Grenzgebiete (3) [Results in Mathematics and Related Areas (3)]. SpringerVerlag, Berlin, 1986.

[15] P. Hornung. Approximation of flat $W^{2,2}$ isometric immersions by smooth ones. Arch. Ration. Mech. Anal., 199(3):1015-1067, 2011.

[16] P. Hornung. Fine level set structure of flat isometric immersions. Arch. Ration. Mech. Anal., 199(3):943-1014, 2011.

[17] P. Isett. A proof of Onsager's conjecture. arXiv preprint arXiv:1608.08301, 2016.

[18] R. V. Kohn and H.-M. Nguyen. Analysis of a compressed thin film bonded to a compliant substrate: the energy scaling law. J. Nonlinear Sci., 23(3):343-362, 2013.

[19] E. M. Kramer and T. A. Witten. Stress condensation in crushed elastic manifolds. Phys. Rev. Lett., 78:1303-1306, Feb 1997.

[20] N. H. Kuiper. On $C^{1}$-isometric imbeddings. I, II. Nederl. Akad. Wetensch. Proc. Ser. A. 58, Indag. Math., 17:545-556, 683-689, 1955.

[21] M. Lewicka, M. G. Mora, and M. R. Pakzad. Shell theories arising as low energy $\Gamma-$ limit of 3d nonlinear elasticity. Ann. Sc. Norm. Super. Pisa Cl. Sci. (5), 9(2):253-295, 2010 .

[22] M. Lewicka and M. R. Pakzad. Convex integration for the Monge-Ampère equation in two dimensions. ArXiv e-prints, August 2015.

[23] J. Lidmar, L. Mirny, and D. R. Nelson. Virus shapes and buckling transitions in spherical shells. Physical Review E, 68(5):051910, 2003.

[24] A. Lobkovsky, S. Gentges, H. Li, D. Morse, and T. A. Witten. Scaling properties of stretching ridges in a crumpled elastic sheet. Science, 270(5241):1482-1485, 1995.

[25] A. E. Lobkovsky. Boundary layer analysis of the ridge singularity in a thin plate. Phys. Rev. E, 53:3750-3759, April 1996.

[26] A. E. Lobkovsky and T. A. Witten. Properties of ridges in elastic membranes. Phys. Rev. E, 55:1577-1589, February 1997.

[27] S. Müller. Higher integrability of determinants and weak convergence in $L^{1}$. J. Reine Angew. Math., 412:20-34, 1990.

[28] S. Müller and H. Olbermann. Almost conical deformations of thin sheets with rotational symmetry. SIAM J. Math. Anal., 46(1):25-44, 2014.

[29] S. Müller and H. Olbermann. Conical singularities in thin elastic sheets. Calc. Var. Partial Differential Equations, 49(3-4):1177-1186, 2014.

[30] J. Nash. $C^{1}$ isometric imbeddings. Ann. of Math. (2), 60:383-396, 1954.

[31] H. Olbermann. Energy scaling law for the regular cone. J. Nonlinear Sci., 26:287-314, 2016.

[32] H. Olbermann. Energy scaling law for a single disclination in a thin elastic sheet. Arch. Rat. Mech. Anal., 2017. DOI 10.1007/s00205-017-1093-4.

[33] M. R. Pakzad. On the Sobolev space of isometric immersions. J. Differential Geom., 66(1):47-69, 2004.

[34] A. V. Pogorelov. Extrinsic geometry of convex surfaces. American Mathematical Society, Providence, R.I., 1973. Translated from the Russian by Israel Program for Scientific Translations, Translations of Mathematical Monographs, Vol. 35. 
[35] A. E. Romanov. Mechanics and physics of disclinations in solids. Eur. J. Mech. A Solids, 22(5):727-741, 2003. 5th EUROMECH Solid Mechanics Conference (Thessaloniki, 2003).

[36] H. S. Seung and David R. Nelson. Defects in flexible membranes with crystalline order. Phys. Rev. A, 38:1005-1018, Jul 1988.

[37] S. C. Venkataramani. Lower bounds for the energy in a crumpled elastic sheet - a minimal ridge. Nonlinearity, 17(1):301-312, 2004.

[38] T. A. Witten. Stress focusing in elastic sheets. Rev. Mod. Phys., 79:643-675, Apr 2007.

[39] A. Yavari and A. Goriely. Riemann-Cartan geometry of nonlinear disclination mechanics. Math. Mech. Solids, 18(1):91-102, 2013.

(Heiner Olbermann) Universität Leipzig, Germany

E-mail address: heiner.olbermann@math.uni-leipzig.de 